doi: $10.2306 /$ scienceasia1513-1874.2013.39.306

\title{
Global convergence of two spectral conjugate gradient methods
}

\author{
Mahdi Ghanbari, Tahir Ahmad*, Norma Alias, Mohammadreza Askaripour \\ Ibnu Sina Institute for Fundamental Science Studies, Universiti Teknologi Malaysia, 81310 Skudai, Johor, \\ Malaysia
}

${ }^{*}$ Corresponding author, e-mail: tahir@ibnusina.utm.my

Received 19 Dec 2012

Accepted 8 May 2013

\begin{abstract}
Two new nonlinear spectral conjugate gradient methods for solving unconstrained optimization problems are proposed. One is based on the Hestenes and Stiefel (HS) method and the spectral conjugate gradient method. The other is based on a mixed spectral HS-CD conjugate gradient method, which combines the advantages of the spectral conjugate gradient method, the HS method, and the CD method. The directions generated by the methods are descent directions for the objective function. Under mild conditions, we prove that the spectral conjugate gradient methods with an Armijo-type line search are globally convergent. Numerical results show the proposed methods are promising.
\end{abstract}

KEYWORDS: unconstrained optimization, inexact line search

\section{INTRODUCTION}

Unconstrained optimization problems have extensive applications, for example, in petroleum exploration, aerospace, and transportation ${ }^{1-3}$. The purpose of this paper is to study the global convergence properties and practical computational performance of two Hestenes and Stiefel (HS) spectral conjugate gradient methods for unconstrained optimization without restarts, and with suitable conditions.

Consider the following unconstrained optimization problem:

$$
\min _{x \in R^{n}} f(x)
$$

where $f: R^{n} \rightarrow R$ is continuously differentiable and its gradient is available. Iterative methods are widely used and the iterative formula is given by

$$
x_{k+1}=x_{k}+\alpha_{k} d_{k}
$$

where $x_{k} \in R^{n}$ is the $k$ th approximation to the solution, $\alpha_{k}$ is a positive scalar and called the stepsize which is determined by some line search and $d_{k}$ is a search direction. There are many kinds of iterative methods that include Newton method ${ }^{4,5}$, steepest descent $\operatorname{method}^{6}$, and nonlinear conjugate gradient $\operatorname{method}^{7,8}$. The conjugate gradient methods are the most famous methods for solving (1), especially in the case of large scale optimization problems in scientific and engineering computation due to the simplicity of their iteration and low memory requirements. The search direction $d_{k}$ is defined by

$$
d_{k}= \begin{cases}-g_{k}, & k=1 \\ -g_{k}+\beta_{k} d_{k-1}, & k \geqslant 2\end{cases}
$$

where $g_{k}=\nabla f\left(x_{k}\right)$ and $\beta_{k}$ is a scalar which determines the different conjugate methods. The wellknown formulae for $\beta_{k}$ such as $\beta_{k}^{\mathrm{HS} 9}, \beta_{k}^{\mathrm{FR} \mathrm{10}}, \beta_{k}^{\mathrm{PRP} 11}$, $\beta_{k}^{\mathrm{CD} 12}, \beta_{k}^{\mathrm{LS} 13}, \beta_{k}^{\mathrm{DY} 14}$ can be found in many related papers. The convergence behaviour of conjugate gradient methods with these formulae under some different line search conditions has been widely studied by many authors (see Refs. 12-15). In the original HS method proposed by Hestenes and Stiefel ${ }^{9} \beta_{k}$ is defined by

$$
\beta_{k}^{\mathrm{HS}}=\frac{g_{k}^{\mathrm{T}} y_{k}}{y_{k}^{\mathrm{T}} d_{k-1}},
$$

where $y_{k}=g_{k}-g_{k-1}$. In practical computation, the HS method is generally believed to be one of the most efficient conjugate gradient methods. Recently, some modified HS formulae have been proposed ${ }^{16-18}$. In these methods, the search direction is constructed to possess the sufficient descent property, and the theory of global convergence is established with different line search strategies. Zhang ${ }^{17}$ proposed a three-term HS conjugate gradient method (called TTHS), in which the direction $d_{k}$ is given by

$$
d_{k}=-g_{k}+\beta_{k}^{\mathrm{HS}} d_{k-1}+\theta_{k} y_{k-1}, \theta_{k}=\frac{g_{k}^{\mathrm{T}} d_{k-1}}{d_{k-1}^{\mathrm{T}} y_{k-1}} .
$$


Fletcher ${ }^{12}$ proposed the $\mathrm{CD}$ method, in which $\beta_{k}$ is defined by

$$
\beta_{k}^{\mathrm{CD}}=-\frac{\left\|g_{k}\right\|^{2}}{d_{k-1}^{\mathrm{T}} g_{k-1}},
$$

where $\|$.$\| denotes the Euclidean norm of vectors.$ An important property of the CD method is that the method will produce a descent direction under the strong Wolfe line search:

$$
\begin{gathered}
f\left(x_{k}+\alpha_{k} d_{k}\right) \leqslant f\left(x_{k}\right)+\delta \alpha_{k} g_{k}^{\mathrm{T}} d_{k}, \\
d_{k}^{\mathrm{T}} g\left(x_{k}+\alpha_{k} d_{k}\right) \geqslant \sigma d_{k}^{\mathrm{T}} g_{k},
\end{gathered}
$$

where $0<\delta<\sigma<1$. Some good results from the $\mathrm{CD}$ method have also been reported in recent years ${ }^{19-21}$. Another popular method of solving problem (1) is the spectral gradient method, which was developed originally by Barzilai and Borwein ${ }^{22}$. Ray$\mathrm{dan}^{23}$ further introduced the spectral gradient method for potentially large-scale unconstrained optimization problems. Recently, Birgin and Martinez ${ }^{24}$ proposed a spectral conjugate gradient method by combining the conjugate gradient method and spectral gradient method. The direction $d_{k}$ is given by

$$
d_{k}=-\theta_{k} g_{k}+\beta_{k} s_{k-1}
$$

where $s_{k}=x_{k}-x_{k-1}$ and

$$
\begin{aligned}
\beta_{k}^{\mathrm{SP}} & =\frac{\left(\theta_{k} y_{k-1}-s_{k-1}\right)^{\mathrm{T}} g_{k}}{s_{k-1}^{\mathrm{T}} y_{k-1}}, \\
\beta_{k}^{\mathrm{SPR}} & =\frac{\theta_{k} y_{k-1}^{\mathrm{T}} g_{k}}{\alpha_{k} \theta_{k-1} g_{k-1}^{\mathrm{T}} g_{k-1}}, \\
\beta_{k}^{\mathrm{SFR}} & =\frac{\theta_{k} g_{k}^{\mathrm{T}} g_{k}}{\alpha_{k} \theta_{k-1} g_{k-1}^{\mathrm{T}} g_{k-1}},
\end{aligned}
$$

and $\theta_{k}$ is taken to be the spectral gradient and is computed from

$$
\theta_{k}=\frac{s_{k-1}^{\mathrm{T}} s_{k-1}}{s_{k-1}^{\mathrm{T}} y_{k-1}} .
$$

The numerical results show that these methods are very effective. Unfortunately, the spectral conjugate gradient method ${ }^{24}$ cannot guarantee to generate descent directions. Hence, based on the FR formula, Zhang et $\mathrm{al}^{25}$ modified the FR method so that the direction generated is always a descent direction. The $d_{k}$ is defined by the following

$$
d_{k}= \begin{cases}-g_{k}, & k=1, \\ -\theta_{k} g_{k}+\beta_{k}^{\mathrm{FR}} d_{k-1}, & k \geqslant 2,\end{cases}
$$

where $\theta_{k}=\left(d_{k-1}^{\mathrm{T}} y_{k-1}\right) /\left(\left\|g_{k-1}\right\|^{2}\right)$. They proved that this method can guarantee to generate descent directions and is globally convergent. In this paper, motivated by the success of the spectral gradient method, we first propose a new spectral conjugate gradient method by combining the HS method and the spectral gradient method. The direction is given by (3) and

$$
\begin{gathered}
\beta_{k}= \begin{cases}\beta_{k}^{\mathrm{HS}}, & g_{k}^{\mathrm{T}} d_{k-1}>0, \\
0, & \text { otherwise },\end{cases} \\
\theta_{k}=1-\frac{\left|g_{k}^{\mathrm{T}} d_{k-1}\right|}{g_{k-1}^{\mathrm{T}} d_{k-1}} .
\end{gathered}
$$

Then, another new spectral conjugate gradient method obtained by combining the HS method and CD is proposed. The direction is given by (3) and

$$
\begin{gathered}
\beta_{k}= \begin{cases}\beta_{k}^{\mathrm{HS}}, & g_{k}^{\mathrm{T}} d_{k-1}>0, \\
\beta_{k}^{\mathrm{CD}}, & g_{k}^{\mathrm{T}} d_{k-1} \leqslant 0 .\end{cases} \\
\theta_{k}=1-\frac{g_{k}^{\mathrm{T}} d_{k-1}}{g_{k-1}^{\mathrm{T}} d_{k-1}} .
\end{gathered}
$$

Under some mild conditions, we give the global convergence of the new spectral conjugate gradient methods with an Armijo-type line search ${ }^{26}$. The rest of this paper is organized as follows. First, we propose the corresponding algorithms and give some assumptions and lemmas, which are usually used in the proof of the global convergence properties of nonlinear conjugate gradient methods. Then, the global convergence of the new spectral conjugate methods will be proven. Some numerical experiments will be done to test the efficiency, especially in comparison with the modified $\mathrm{FR}^{25}$ and the spectral PRP methods ${ }^{26}$ in the last part of the paper.

\section{ALGORITHMS AND LEMMAS}

In this section, we will give the following assumption on objective function, which have often been used in the literature to analyse the global convergence of nonlinear conjugate gradient method and the spectral conjugate gradient method with inexact line searches.

Assumption 1: the level set $\Omega=\{x \mid f(x) \leqslant$ $\left.f\left(x_{1}\right)\right\}$ is bounded, where $x_{1}$ is the starting point.

Assumption 2: in some neighbourhood $N$ of $\Omega$, the objective function is continuously differentiable and its gradient is Lipschitz continuous, namely, there exists a constant $L>0$ such that

$$
\|g(x)-g(y)\| \leqslant L\|x-y\| \text { for } x, y \in N .
$$

Now we present the new spectral conjugate gradient method. 
Algorithm 1 (Spectral HS Conjugate Gradient)

Step 1: Given constant $\delta_{1}, \rho \in(0,1), \delta_{2}>0, \epsilon>0$.

Choose an initial point $x_{1} \in R^{n}$, let $k=1$.

Step 2: If $\left\|g_{k}\right\| \leqslant \epsilon$, then the algorithm stops. Otherwise, compute $d_{k}$ by (3), (14) and $\beta_{k}$ by (13).

Step 3: Compute step-size $\alpha_{k}=\max \left\{\rho^{j}, j=\right.$ $0,1,2, \ldots\}$ such that

$$
f\left(x_{k}+\alpha_{k} d_{k}\right) \leqslant f\left(x_{k}\right)+\delta_{1} \alpha_{k} g_{k}^{\mathrm{T}} d_{k}-\delta_{2} \alpha_{k}^{2}\left\|d_{k}\right\|^{2}
$$

Step 4: Set $x_{k+1}=x_{k}+\alpha_{k} d_{k}$, and $k=k+1$. Return to Step 2.

Algorithm 2 (Spectral HS-CD Conjugate Gradient) Whole steps of the spectral HS-CD algorithm are defined as Algorithm 1 except $d_{k}$ and $\beta_{k}$ in Step 2 that are computed by (3), (16) and (15), respectively.

The following theorem shows that both Algorithms 1 and 2 possess a descent direction in each iteration.

Theorem 1 Let the sequences $g_{k}$ and $d_{k}$ be generated by Algorithms 1 and 2 and let the step-size $\alpha_{k}$ be determined by any line search, then

$$
g_{k}^{\mathrm{T}} d_{k}<0 .
$$

Proof: We can prove the conclusion by induction. From $\left\|g_{1}\right\|^{2}=-g_{1}^{\mathrm{T}} d_{1}$, the conclusion (19) holds for $k=1$. Now we assume that the conclusion is true for $k-1$ and $g_{k} \neq 0$, that is $g_{k-1}^{\mathrm{T}} d_{k-1}<0$. In the following, we need to prove that the conclusion holds for $k$. If $g_{k}^{\mathrm{T}} d_{k-1}>0$, then, from both Algorithms 1 and $2 \beta_{k}=\beta_{k}^{\mathrm{HS}}$. From (4), (3), (14), (16) and our assumption $g_{k-1}^{\mathrm{T}} d_{k-1}<0$ we have

$$
\begin{aligned}
g_{k}^{\mathrm{T}} d_{k}=- & \left(1-\frac{g_{k}^{\mathrm{T}} d_{k-1}}{g_{k-1}^{\mathrm{T}} d_{k-1}}\right)\left\|g_{k}\right\|^{2} \\
& +\frac{g_{k}^{\mathrm{T}}\left(g_{k}-g_{k-1}\right)}{\left(g_{k}-g_{k-1}\right)^{\mathrm{T}} d_{k-1}} g_{k}^{\mathrm{T}} d_{k-1} \\
=- & \left\|g_{k}\right\|^{2}+\frac{g_{k}^{\mathrm{T}} d_{k-1}}{g_{k-1}^{\mathrm{T}} d_{k-1}} g_{k}^{\mathrm{T}} g_{k} \\
& +\frac{g_{k}^{\mathrm{T}}\left(g_{k}-g_{k-1}\right)}{\left(g_{k}-g_{k-1}\right)^{\mathrm{T}} d_{k-1}} g_{k}^{\mathrm{T}} d_{k-1} \\
=- & \frac{d_{k-1}^{\mathrm{T}}\left(g_{k}-g_{k-1}\right)}{\left(g_{k}-g_{k-1}\right)^{\mathrm{T}} d_{k-1}}\left\|g_{k}\right\|^{2} \\
& +\frac{g_{k}^{\mathrm{T}} d_{k-1}}{g_{k-1}^{\mathrm{T}} d_{k-1}} g_{k}^{\mathrm{T}} g_{k} \\
& +\frac{g_{k}^{\mathrm{T}}\left(g_{k}-g_{k-1}\right)}{\left(g_{k}-g_{k-1}\right)^{\mathrm{T}} d_{k-1}} g_{k}^{\mathrm{T}} d_{k-1} \\
= & \frac{g_{k}^{\mathrm{T}} d_{k-1}}{g_{k-1}^{\mathrm{T}} d_{k-1}} g_{k}^{\mathrm{T}} g_{k}<0 .
\end{aligned}
$$

If $g_{k}^{\mathrm{T}} d_{k-1} \leqslant 0$ then from Algorithm $2 \beta_{k}=\beta_{k}^{\mathrm{CD}}$. From (6), (3), and (16), we have

$$
\begin{aligned}
g_{k}^{\mathrm{T}} d_{k} & =-\theta_{k}\left\|g_{k}\right\|^{2}+\beta_{k}^{\mathrm{CD}} g_{k}^{\mathrm{T}} d_{k-1} \\
& =-\left[1-\frac{g_{k}^{\mathrm{T}} d_{k-1}}{g_{k-1}^{\mathrm{T}} d_{k-1}}\right]\left\|g_{k}\right\|^{2}-\frac{\left\|g_{k}\right\|^{2} g_{k}^{\mathrm{T}} d_{k-1}}{d_{k-1}^{\mathrm{T}} g_{k-1}} \\
& =-\left\|g_{k}\right\|^{2}<0 .
\end{aligned}
$$

If $g_{k}^{\mathrm{T}} d_{k-1} \leqslant 0$ then from Algorithm $1 \beta_{k}=0$. From (3) and (14), we have

$$
\begin{aligned}
g_{k}^{\mathrm{T}} d_{k} & =-\theta_{k}\left\|g_{k}\right\|^{2}=-\left(1+\frac{g_{k}^{\mathrm{T}} d_{k-1}}{g_{k-1}^{\mathrm{T}} d_{k-1}}\right)\left\|g_{k}\right\|^{2} \\
& =-\left\|g_{k}\right\|^{2}-\frac{g_{k}^{\mathrm{T}} d_{k-1}}{g_{k-1}^{\mathrm{T}} d_{k-1}}\left\|g_{k}\right\|^{2} \\
& \leqslant-\left\|g_{k}\right\|^{2}<0
\end{aligned}
$$

From (20), (21) and (22), we know that the conclusion (19) holds for all $k$.

Proposition 1 (See Ref. 26). Let $f: R^{n} \rightarrow R$ be a continuously differentiable function. Suppose that $d$ is a descent direction of $f$ at $x$. Then there exists $J_{0}$ such that

$$
f(x+\alpha d) \leqslant f(x)+\delta_{1} \alpha g^{\mathrm{T}} d-\delta_{2} \alpha^{2}\|d\|^{2},
$$

where $\alpha=\rho^{J_{0}}, g$ is the gradient vector of $f$ at $x, \delta_{1}$, $\rho \in(0,1), \delta_{2}>0$ are given constant scalars.

Remark 1 From Proposition 1, it is known that both Algorithms 1 and 2 are well defined. In addition, it is easy to see that a larger descent magnitude can be obtained at each step by the modified Armijo-type line search (18). The following conclusion is given in Lemma 3.3 of Ref. 26.

Lemma 1 With Assumptions 1 and 2 there exists a constant $m>0$ such that

$$
\alpha_{k}>m \frac{\left|g_{k}^{\mathrm{T}} d_{k}\right|}{\left\|d_{k}\right\|^{2}},
$$

holds for all sufficiently large $k$.

Lemma 2 Under Assumptions 1 and 2,

$$
\begin{aligned}
& \sum_{k \geqslant 1} \frac{\left(g_{k}^{\mathrm{T}} d_{k}\right)^{2}}{\left\|g_{k}\right\|^{2}}<\infty, \\
& \lim _{k \rightarrow \infty} \alpha_{k}^{2}\left\|d_{k}\right\|^{2}=0 .
\end{aligned}
$$


Proof: From the line search rule (18) and Assumption 1, there exists a constant $M$ such that

$$
\begin{aligned}
& \sum_{k=0}^{n-1}-\delta_{1} \alpha_{k} g_{k}^{\mathrm{T}} d_{k}+\delta_{2} \alpha_{k}^{2}\left\|d_{k}\right\|^{2} \\
& \leqslant \sum_{k=0}^{n-1}\left(f\left(x_{k}\right)-f\left(x_{k+1}\right)\right)<2 M .
\end{aligned}
$$

Then from Lemma 1 we have

$$
\begin{aligned}
2 M & \geqslant \sum_{k=0}^{n-1}\left(-\delta_{1} \alpha_{k} g_{k}^{\mathrm{T}} d_{k}+\delta_{2} \alpha_{k}^{2}\left\|d_{k}\right\|^{2}\right) \\
& \geqslant \sum_{k=0}^{n-1}\left(\delta_{1} m \frac{\left(g_{k}^{\mathrm{T}} d_{k}\right)^{2}}{\left\|d_{k}\right\|^{2}}+\delta_{2} m^{2} \frac{\left(g_{k}^{\mathrm{T}} d_{k}\right)^{2}}{\left\|d_{k}\right\|^{4}}\left\|d_{k}\right\|^{2}\right) \\
& \geqslant \sum_{k=0}^{n-1}\left(\delta_{1} m+\delta_{2} m^{2}\right) \frac{\left(g_{k}^{\mathrm{T}} d_{k}\right)^{2}}{\left\|d_{k}\right\|^{2}} .
\end{aligned}
$$

Hence the first conclusion is proven. Since

$$
\begin{aligned}
2 M & \geqslant \sum_{k=0}^{n-1}\left(-\delta_{1} \alpha_{k} g_{k}^{\mathrm{T}} d_{k}+\delta_{2} \alpha_{k}^{2}\left\|d_{k}\right\|^{2}\right) \\
& \geqslant \delta_{2} \sum_{k=0}^{n-1} \alpha_{k}^{2}\left\|d_{k}\right\|^{2} .
\end{aligned}
$$

The series $\sum_{k=0}^{n-1} \alpha_{k}^{2}\left\|d_{k}\right\|^{2}$ is convergent. Thus $\lim _{k \rightarrow 0} \alpha_{k}^{2}\left\|d_{k}\right\|^{2}=0$. The second conclusion is obtained.

Lemma 3 Suppose that Assumptions 1 and 2 hold. Consider both Algorithms 1 and 2, where $\alpha_{k}$ is obtain by the modified Armijo line search (18). If there exists a constant $\epsilon>0$ such that for all $k>0,\left\|g_{k}\right\|>\epsilon$. Then there exists a sufficiently large number $k_{0}$ such that for $k \geqslant k_{0}$, the scalars $\beta_{k}$ in Algorithms 1 and 2 satisfy

$$
\left|\beta_{k}\right| \leqslant\left|\frac{g_{k}^{\mathrm{T}} d_{k}}{g_{k-1}^{\mathrm{T}} d_{k-1}}\right| .
$$

Proof: If $g_{k}^{\mathrm{T}} d_{k-1}>0$, from (2), (3), (17) and using the Cauchy-Schwarz inequality, we have

$$
\begin{aligned}
\left|\beta_{k}\right| & =\left|\frac{g_{k}^{\mathrm{T}}\left(g_{k}-g_{k-1}\right)}{\left(g_{k}-g_{k-1}\right)^{\mathrm{T}} d_{k-1}}\right| \\
& \leqslant \frac{\left|g_{k}^{\mathrm{T}}\left(g_{k}-g_{k-1}\right)\right|}{\left|-g_{k-1}^{\mathrm{T}} d_{k-1}\right|} \\
& \leqslant \frac{\left\|g_{k}\right\|\left\|g_{k}-g_{k-1}\right\|}{\left|g_{k-1}^{\mathrm{T}} d_{k-1}\right|} \\
& \leqslant \frac{\left\|g_{k}\right\| L \alpha_{k-1}\left\|d_{k-1}\right\|}{\left|g_{k-1}^{\mathrm{T}} d_{k-1}\right| .}
\end{aligned}
$$

From (26) in Lemma 2, it follows that

$$
\lim _{k \rightarrow \infty} \alpha_{k-1}^{2}\left\|d_{k-1}\right\|^{2}=0 .
$$

In particular, we have

$$
\lim _{k \rightarrow \infty} \alpha_{k-1}\left\|d_{k-1}\right\|=0 .
$$

Thus there exists a sufficient large number $k_{0}$ such that for $k \geqslant k_{0}$,

$$
0 \leqslant \alpha_{k-1}\left\|d_{k-1}\right\|<\frac{\left|g_{k}^{\mathrm{T}} d_{k}\right|}{\left\|g_{k}\right\| L} .
$$

Hence by substituting (34) in (31), we have (30).

If $g_{k}^{\mathrm{T}} d_{k-1} \leqslant 0$, then from (6), (15), (13) and (21), we have (30).

\section{GLOBAL CONVERGENCE PROPERTY}

Theorem 2 Under Assumptions 1 and 2,

$$
\liminf _{k \rightarrow \infty}\left\|g_{k}\right\|=0 .
$$

Proof: Suppose that there exists a positive constant $\epsilon>0$ such that $\left\|g_{k}\right\|>\epsilon$ for all $k$. From (3), it follows that

$$
\begin{aligned}
& \left\|g_{k}\right\|^{2}=\left(-\theta_{k} g_{k}+\beta_{k} d_{k-1}\right)^{\mathrm{T}}\left(-\theta_{k} g_{k}+\beta_{k} d_{k-1}\right) \\
& \quad=\theta_{k}^{2}\left\|g_{k}\right\|^{2}-2 \theta_{k} \beta_{k} d_{k-1}^{\mathrm{T}} g_{k}+\beta_{k}^{2}\left\|d_{k-1}\right\|^{2} \\
& =\theta_{k}^{2}\left\|g_{k}\right\|^{2}-2 \theta_{k}\left(d_{k}^{\mathrm{T}}+\theta_{k} g_{k}^{\mathrm{T}}\right) g_{k}+\beta_{k}^{2}\left\|d_{k-1}\right\|^{2} \\
& =\theta_{k}^{2}\left\|g_{k}\right\|^{2}-2 \theta_{k} d_{k}^{\mathrm{T}} g_{k}-2 \theta_{k}^{2}\left\|g_{k}\right\|^{2}+\beta_{k}^{2}\left\|d_{k-1}\right\|^{2} \\
& =\beta_{k}^{2}\left\|d_{k-1}\right\|^{2}-2 \theta_{k} d_{k}^{\mathrm{T}} g_{k}-\theta_{k}^{2}\left\|g_{k}\right\|^{2} .
\end{aligned}
$$

Dividing (36) by $\left(g_{k}^{\mathrm{T}} d_{k}\right)^{2}$ then from Lemma 3, there exists a sufficient large $k_{0}$ such that for $k \geqslant k_{0}$, we obtain

$$
\begin{aligned}
& \frac{\left\|d_{k}\right\|^{2}}{\left(g_{k}^{\mathrm{T}} d_{k}\right)^{2}}=\frac{\beta_{k}^{2}\left\|d_{k-1}\right\|^{2}-2 \theta_{k} d_{k}^{\mathrm{T}} g_{k}-\theta_{k}^{2}\left\|g_{k}\right\|^{2}}{\left(g_{k}^{\mathrm{T}} d_{k}\right)^{2}} \\
& \quad \leqslant \frac{\left(g_{k}^{\mathrm{T}} d_{k}\right)^{2}}{\left(g_{k-1}^{\mathrm{T}} d_{k-1}\right)^{2}} \frac{\left\|d_{k-1}\right\|^{2}}{\left(g_{k}^{\mathrm{T}} d_{k}\right)^{2}}-\frac{2 \theta_{k}}{g_{k}^{\mathrm{T}} d_{k}}-\theta_{k}^{2} \frac{\left\|g_{k}\right\|^{2}}{\left(g_{k}^{\mathrm{T}} d_{k}\right)^{2}} \\
& \quad=\frac{\left\|d_{k-1}\right\|^{2}}{\left(g_{k-1}^{\mathrm{T}} d_{k-1}\right)^{2}}-\left(\theta_{k} \frac{\left\|g_{k}\right\|}{g_{k}^{\mathrm{T}} d_{k}}+\frac{1}{\left\|g_{k}\right\|}\right)^{2}+\frac{1}{\left\|g_{k}\right\|^{2}} \\
& \quad \leqslant \frac{\left\|d_{k-1}\right\|^{2}}{\left(g_{k-1}^{\mathrm{T}} d_{k-1}\right)^{2}}+\frac{1}{\left\|g_{k}\right\|^{2}} .
\end{aligned}
$$


Table 1 The numerical results of the MFR (M), SHS-CD (C), SHS (S), and SPRP (P) methods.

\begin{tabular}{|c|c|c|c|c|c|c|c|c|c|c|c|c|c|}
\hline \multirow[t]{2}{*}{ problems } & \multirow[t]{2}{*}{ Dim } & \multicolumn{4}{|c|}{$\mathrm{GV} / 10^{-6}$} & \multicolumn{4}{|c|}{ number of iterations } & \multicolumn{4}{|c|}{ number of function evaluations } \\
\hline & & $\mathrm{M}$ & $\mathrm{C}$ & S & $\mathrm{P}$ & M & $\mathrm{C}$ & $\mathrm{S}$ & $\mathrm{P}$ & M & $\mathrm{C}$ & $\mathrm{S}$ & $\mathrm{P}$ \\
\hline Brown almost-linear & 100 & 6.54 & 7.60 & 7.60 & 6.54 & 24 & 29 & 29 & 24 & 2161 & 2126 & 2126 & 2161 \\
\hline $\begin{array}{l}\text { Trigonometric } \\
\text { function }\end{array}$ & 100 & 9.99 & 9.87 & 9.64 & 7.24 & 589 & 818 & 239 & 146 & 18959 & 26211 & 1097 & 17151 \\
\hline $\begin{array}{l}\text { Linear function } \\
\text { full rank }\end{array}$ & 100 & 9.78 & 9.78 & 8.28 & 9.78 & 169 & 169 & 89 & 169 & 70 & 70 & 90 & 70 \\
\hline $\begin{array}{l}\text { Linear function } \\
\text { - rank } 1\end{array}$ & 40 & 8.20 & 2.16 & 3.89 & 5.22 & 117 & 117 & 129 & 1499 & 23383 & 23305 & 33553 & 469714 \\
\hline Brown almost-linear & 20 & 9.89 & 7.22 & 9.16 & 7.28 & 1254 & 833 & 1898 & 1680 & 63366 & 33982 & 93890 & 89935 \\
\hline Discrete boundal & 20 & 9.57 & 9.84 & 9.66 & 8.61 & 1714 & 1769 & 7899 & 2699 & 38461 & 47241 & 166750 & 50001 \\
\hline Linear function & 20 & 2.54 & 9.88 & 8.58 & 9.29 & 228 & 168 & 177 & 198 & 34273 & 25177 & 26394 & 29728 \\
\hline Penalty function II & 20 & 5.91 & 6.97 & 5.65 & 9.23 & 250 & 899 & 699 & 4499 & 6948 & 51848 & 22771 & 140659 \\
\hline $\begin{array}{l}\text { Linear function } \\
\text { full rank }\end{array}$ & 12 & 3.66 & 1.47 & 1.64 & 1.68 & 8 & 11 & 11 & 6 & 97 & 73 & 99 & 25 \\
\hline $\begin{array}{l}\text { Linear function } \\
\text { - rank } 1\end{array}$ & 10 & 7.40 & 1.29 & 2.64 & 9.48 & 42 & 44 & 35 & 74 & 4789 & 4843 & 3777 & 3026 \\
\hline Broyden tridiagonal & 9 & 4.26 & 1.69 & 6.82 & 6.87 & 109 & 59 & 54 & 32 & 2125 & 2319 & 2121 & 6380 \\
\hline Variably dimensioned & 8 & 1.33 & 1.47 & 2.32 & 4.25 & 45 & 21 & 21 & 21 & 1163 & 802 & 774 & 506 \\
\hline $\begin{array}{l}\text { Extended power } \\
\text { singular }\end{array}$ & 8 & 9.76 & 9.74 & 3.84 & 2.57 & 846 & 526 & 9999 & 7899 & 10199 & 5643 & 362113 & 267924 \\
\hline Biggs EXP6 function & 6 & 6.30 & 3.98 & 6.08 & 9.31 & 20999 & 19999 & 31999 & 31899 & 975041 & 796701 & 277479 & 238730 \\
\hline Penalty fu & 5 & 4.45 & 4.45 & 9.56 & 10.00 & 11999 & 11999 & 8999 & 6486 & 333012 & 333012 & 22596 & 6530 \\
\hline Broyd & 4 & 9.93 & 1.24 & 9.16 & 7.14 & 119 & 42 & 37 & 43 & 2864 & 783 & 694 & 816 \\
\hline $\begin{array}{l}\text { Extended power } \\
\text { singular }\end{array}$ & 4 & 9.23 & 9.98 & 9.31 & 6.93 & 863 & 406 & 5989 & 3899 & 10199 & 6030 & 216981 & 13206 \\
\hline Brown almost-linear & 4 & 7.06 & 9.77 & 8.53 & 4.70 & 122 & 121 & 168 & 120 & 249 & 283 & 1984 & 1379 \\
\hline $\begin{array}{l}\text { Discrete boundary } \\
\text { value }\end{array}$ & 4 & 9.42 & 4.21 & 7.23 & 7.70 & 35 & 27 & 39 & 32 & 724 & 561 & 771 & 843 \\
\hline Powell singular & 4 & 9.60 & 9.18 & 5.49 & 7.79 & 667 & 408 & 679 & 2369 & 10824 & 6030 & 24736 & 80201 \\
\hline Brov & 4 & & .45 & 2.56 & 9.7 & 239 & 262 & 262 & 338 & 791 & 519 & 882 & 281 \\
\hline Wood func & 4 & 9.71 & 8.05 & 7.49 & 3.41 & 292 & 198 & 254 & 209 & 18669 & 11208 & 14688 & 270532 \\
\hline $\begin{array}{l}\text { Kowalik and } \\
\text { Osboren function }\end{array}$ & 4 & 6.92 & 7.80 & 8.18 & 9.78 & 9995 & 9899 & 2999 & 3044 & 416535 & 415016 & 28014 & 24340 \\
\hline Gauss & 3 & 7.61 & 3.11 & 5.74 & 7.44 & 8 & 6 & 6 & 8 & 101 & 51 & 59 & 107 \\
\hline Bard function & 3 & 9.78 & 9.81 & 6.33 & - & 715 & 614 & 1834 & & 1102 & 590 & 36939 & \\
\hline $\begin{array}{l}\text { Box three } \\
\text {-dimensional }\end{array}$ & 3 & 9.16 & 9.26 & 2.72 & 9.13 & 597 & 579 & 599 & 677 & 1190 & 1100 & 4681 & 1124 \\
\hline Helical & 3 & 9.63 & 8.39 & 5.94 & 9.29 & 19 & 31 & 30 & 19 & 879 & 1493 & 1400 & 2613 \\
\hline $\begin{array}{l}\text { Jennrich } \\
\text { and Sampson }\end{array}$ & 2 & 7.95 & 5.74 & 5.74 & 4.28 & 55 & 20 & 20 & 21 & 2183 & 1129 & 1129 & 882 \\
\hline $\begin{array}{l}\text { Freudenstein } \\
\text { and Roth }\end{array}$ & 2 & 1.13 & 2.92 & 3.27 & 2.65 & 137 & 37 & 56 & 68 & 4739 & 1010 & 1461 & 704 \\
\hline Rosenbrock & 2 & 4.98 & 1.68 & 9.45 & 3.55 & 238 & 66 & 175 & 106 & 2598 & 1824 & 1639 & 1073 \\
\hline Beal & 2 & 7.56 & 5.51 & 2.93 & 6.93 & 74 & 39 & 45 & 45 & 1920 & 1165 & 1063 & 1263 \\
\hline $\begin{array}{l}\text { Brown badly } \\
\text { scan function }\end{array}$ & 2 & - & 6.68 & 6.68 & 9.44 & - & 59 & 59 & 88 & - & 16581 & 16581 & 24285 \\
\hline
\end{tabular}

Dim: the dimension of the objective function; GV: the gradient value of the objective function when the algorithm stops; MFR: the modified FR conjugate gradient method in Ref. 25; SHS-CD: the new spectral HS-CD method presented in this paper; SHS: the new spectral HS method presented in this paper; SPRP: the spectral PRP conjugate gradient method in Ref. 26.

Therefore

$$
\begin{aligned}
\frac{\left\|d_{k}\right\|^{2}}{\left(g_{k}^{\mathrm{T}} d_{k}\right)^{2}} & \leqslant \frac{\left\|d_{k-1}\right\|^{2}}{\left(g_{k-1}^{\mathrm{T}} d_{k-1}\right)^{2}}+\frac{1}{\left\|g_{k}\right\|^{2}} \\
& \leqslant \ldots \leqslant \frac{\left\|d_{k_{0}}\right\|^{2}}{\left(g_{k_{0}}^{\mathrm{T}} d_{k_{0}}\right)}+\sum_{i=k_{0}+1}^{k} \frac{1}{\left\|g_{k}\right\|^{2}} \\
& \leqslant \frac{c_{0}}{\epsilon^{2}}+\sum_{i=k_{0}+1}^{k} \frac{1}{\epsilon^{2}}=\frac{c_{0}+k-k_{0}}{\epsilon^{2}}
\end{aligned}
$$

where $c_{0}=\epsilon^{2}\left\|d_{k_{0}}\right\|^{2} /\left(g_{k_{0}}^{\mathrm{T}} d_{k_{0}}\right)^{2}$ is a nonnegative constant. The last inequality implies

$$
\sum_{k \geqslant 1} \frac{\left(g_{k}^{\mathrm{T}} d_{k}\right)^{2}}{\left\|d_{k}\right\|^{2}} \geqslant \sum_{k \geqslant k_{0}} \frac{\left(g_{k}^{\mathrm{T}} d_{k}\right)^{2}}{\left\|d_{k}\right\|^{2}}>\epsilon^{2} \sum_{k \geqslant k_{0}} \frac{1}{c_{0}+k+k_{0}}
$$

The right-hand side of this is infinite which contradicts the result of Lemma 2. Hence the conclusion (35) holds.

\section{NUMERICAL EXPERIMENTS}

In this section, we report some numerical results. Under the modified Armijo line search (18), we compare the performances of the iteration number and the 
function evaluation number of the new methods with that of modified $\mathrm{FR}^{25}$, spectral $\mathrm{PRP}^{26}$ on the given test problems which come from ${ }^{27}$.

All codes were written in MATLAB 7.0.1 and were implemented on a PC with $2.0 \mathrm{GHz} \mathrm{CPU}, 1 \mathrm{~GB}$ RAM, and Windows 7. The parameters used were $\epsilon=10^{-5}, \rho=0.9, \delta_{1}=0.25, \delta_{2}=0.45$.

Comparison of the results in Table 1 shows that the proposed algorithms in this paper are promising.

\section{CONCLUSIONS}

In this paper, two new spectral HS conjugate gradient algorithms have been developed for solving unconstrained minimization problems. Under some mild conditions, the global convergence has been proven with an Armijo-type line search rule. Compared with the other similar algorithms, the numerical performances of the developed algorithms are promising.

Acknowledgements: The authors would like to thank the school of postgraduate studies of Universiti Teknologi Malaysia for funding this research under the SPS Fellowship grant.

\section{REFERENCES}

1. $\mathrm{Hu} \mathrm{ZZ}, \mathrm{Hu} \mathrm{XY}$, He ZX (2006) Pseudo-threedimensional magnetotelluric inversion with nonlinear conjugate gradients. Chin J Geophys 49, 1226-34.

2. Keane A, Nair P (2005) Computational Approaches for Aerospace Design: The Pursuit of Excellence, Wiley.

3. Xie C, Travis Waller S (2012) Stochastic traffic assignment, Lagrangian dual, and unconstrained convex optimization. Transport Res B 46, 1023-42.

4. Frontini M, Sormani E (2003) Some variants of Newton's method with third-order convergence. Appl Math Comput 140, 419-26.

5. Zban AYO (2004) Some new variants of Newton's method. Appl Math Lett 17, 677-82.

6. Yuan Y (2004) A New Stepsize for the Steepest Descent Method, Research report, Institute of Computational Mathematics and Scientific/Engineering Computing, Academy of Mathematics and Systems Sciences, Chinese Academy of Sciences.

7. Dai YH, Yuan YX (2000) Nonlinear Conjugate Gradient Methods, Shanghai Scientific and Technical Publishers, Shanghai.

8. Nocedal J, Wright SJ (1999) Numerical Optimization, Springer-Verlag, New York.

9. Hestenes MR, Stiefel ER (1964) Methods of conjugate gradients for solving linear systems. J Res Natl Bur Stand 49B, 409-32.

10. Fletcher R, Reeves C (1964) Function minimization by conjugate gradients. Comput J 7, 149-54.

11. Polak B, Ribière G (1969) Note sur la convergence des méthodes de directions conjuguées. Rev Française Inf Rech Opér Ser 3e Ann 16, 35-43.
12. Fletcher R (1987) Practical methods of optimization. In: Unconstrained Optimization vol I, Wiley, New York.

13. Liu YL, Storey CS (1991) Efficient generalized conjugate gradient algorithms. part 1: theory. J Optim Theor Appl 69, 129-37.

14. Dai YH, Yuan Y (2000) A nonlinear conjugate gradient method with a strong global convergence property. SIAM J Optim 10, 177-82.

15. Polyak BT (1969) The conjugate gradient method in extreme problems. USSR Comp Math Math Phys 9, 94-112.

16. Hager WW, Zhang H (2005) A new conjugate gradient method with guaranteed descent and an efficient line search. SIAM J Optim 16, 170-92.

17. Zhang L, Zhou W, Li D (2007) Some descent threeterm conjugate gradient methods and their global convergence. Optim Meth Software 22, 697-711.

18. Dai Z-F (2011) Two modified HS type conjugate gradient methods for unconstrained optimization problems. Nonlin Anal 74, 927-36.

19. Du X-W, Ye L-Q, Xu C-X (2001) Global convergence of a class of unconstrained optimal methods that include the conjugate descent method. J Appl Math 18, 120-2.

20. Dai Y, Yuan Y (1996) Convergence properties of the conjugate descent method. Adv Math 25, 552-62.

21. Pan C-Y, Chen L-P (2007) A class of efficient new descent methods. Acta Math Applicatae Sin 30, 88-98.

22. Barzilai J, Borwein JM (1988) Two-point step size gradient methods. IMA J Numer Anal 8, 141-8.

23. Raydan M (1997) The Barzilai and Borwein gradient method for the large scale unconstrained minimization problem. SIAM J Optim 7, 26-33.

24. Birgin EG, Martínez JM (2001) A spectral conjugate gradient method for unconstrained optimization. Appl Math Optim 43, 117-28.

25. Zhang L, Zhou W, Li D (2006) Global convergence of a modified Fletcher-Reeves conjugate gradient method with Armijo-type line search. Numer Math 104, 561-72.

26. Jiang H, Deng S, Zheng X, Wan Zh (2012) Global convergence of a modified spectral conjugate gradient method. J Appl Math 2012, 641276.

27. More JJ, Garbow BS, Hillstrom KE (1981) Testing unconstrained optimization software. ACM Trans Math Software 7, 17-41. 